\title{
TIBIAL ROTATION ASSESSMENT USING ARTIFICIAL NEURAL NETWORKS
}

\author{
B. Gültekin Çetiner ${ }^{1 *}$, Murat Sarı ${ }^{2}$ \\ 'International University of Sarajevo, Faculty of Engineering and Natural Sciences, \\ Sarajevo, Bosnia \& Herzegovina. cetiner@ius.edu.ba \\ ${ }^{2}$ Pamukkale University, Faculty of Art and Science, Department of Mathematics, \\ 20020, Denizli, Turkey. msari@pau.edu.tr
}

\begin{abstract}
Assessment of the tibial rotations by the conventional approaches is generally difficult. An investigation has been made in this study to assess the tibial motions based on the prediction of the effects of physical factors as well as a portion of tibial measurements by making use of Artificial Neural Networks (ANN). Therefore, this study aimed at the prediction of the relations between several physical factors and tibial motion measurements in terms of Artificial Neural Networks. These factors include gender, age, weight, and height. Data collected for 484 healthy subjects have been analyzed by Artificial Neural Networks. Promising results showed that the ANN has been found to be appropriate for modeling and simulation in the data assessments. The paper gives detailed results regarding the use of ANN for modeling tibial rotations in terms of physical factors. The study shows the feasibility of ANN to predict the behaviour of knee joints.
\end{abstract}

Key Words- Tibial motion, Artificial Neural Networks

\section{INTRODUCTION}

Motion of the knee joint is of great interest and a number of techniques have been used to describe the range of motion of it [1].

Despite all studies in the literature, there are few investigations examining the tibial motions including the internal and external rotations [2]. However, it has been showed that certain knee injuries are caused by an excessive internal tibial rotation or a delayed external tibial rotation [3]. Since external rotation is linked to knee extension, excessive internal rotation during the stance phase of walking or running may delay the natural external rotation as the knee begins to extend. This has the potential to increase torsional joint stresses at the knee or through tibial shaft and in turn cause knee injury [3].

Evaluation of the tibial rotation by clinical examination is generally rather difficult. Despite existence of important studies in the literature, the normal amounts of the rotations of the tibia have not been clearly specified yet. In literature, there are several methods to assess the tibial rotation such as clinical computerized tomography, magnetic resonant imaging, clinical goniometry, gravity goniometry, videotaping, surface curvature, electronic digital inclinometers, roentgengraphs, fluoroscopy and ultrasounography [24], the Measurement of the Vertical Axial Rotation through Tibial Shaft (MVARTS) system [4] etc. 
In addition to their well-known advantages, most of them have many disadvantages such as being expensive, difficulty in use, time consuming and restriction of daily use etc. At this stage, modeling can be bearing in mind in addition to many methods. There are several methods like using statistics at the modeling stage. ANNs which are being used rather frequently in the last decades provide an alternative model for many information-processing procedures. Since the ANN technique is more flexible, assumption-free methodology, and does not require expertise on statistics it has been used for the reliable data processing and consequent interpretations in the current work. ANN as general modeling tools make the prediction process possible for many different patterns based on the existing data regarding a problem area by finding the hidden relations between the inputs and outputs among the data patterns. They simply learn these hidden relations from the known examples.

This study is mainly to analyze the feasibility of ANN technique to predict tibial rotations based on the physical factors such as age, weight, and height.

\section{METHODS}

\subsection{Participants and Study Design}

Four hundred and eighty four healthy subjects from Kütahya region of Turkey were included in this study. All gave their informed consent for participation. Each participant was informed about test equipment and procedures and was examined individually in a test room by a trained physical therapist who had at least three years experiences in physical therapy. All physical factors about the sample were collected before testing included socio-demographic data including personal information. Considering the collection, the right and left knee internal and external tibial rotations were evaluated.

All subjects participated to this work had no any musculoskeletal, neurological or systemic disease that might have an effect on testing. To assess the tibial rotations based on the gender, weight, age, height (GAWH) information, the subjects who hold the inclusion criteria were studied. The data includes 238 females and 246 males making a total of 484 patterns.

A clinical system of Measuring Vertical Axial Rotation through Tibial Shaft

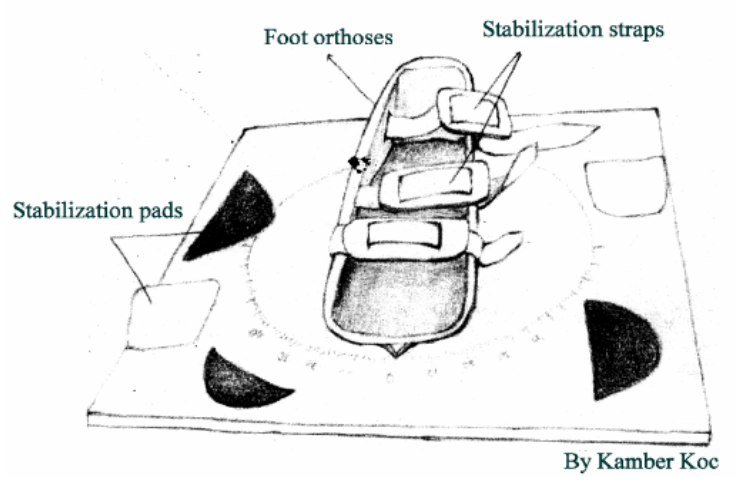

Figure 1. Apparatus of MVARTS system (MVARTS) as described in [4] was used to determine the amount of the passive vertical axial tibial rotations. The MVARTS apparatus adopted for collecting data comprised of a foot orthosis with stabilization pads and a surface table with fixed clinical goniometry as shown in Figure 1. Each healthy subject was tested in a sitting position on an armless chair. The knee is at the position 90 degrees of flexion and the foot is placed in the foot orthosis, which is a part in the apparatus (Figure 2). The evaluators measured the 
tibial external and internal rotations passively and the score was recorded in degree (Figure 3 and Figure 4).

The tibial rotations were also measured radiologically on the X-Rays. The subjects' lower limb was just placed as aforementioned in the system. In order to show geometrical models and the points of the references, four points of the references were placed on the lower limb ( 2 points on the femur and 2 points on the external and internal malleolus of the ankle joint) (Figure 2). Radiological cassette holder was placed under the surface table of the MVARTS system. We exposed during the rotation both the tibia and foot anterior-posterior. The angle of the rotation was calculated on the X-Ray film (Figure 5).

Four measurements related to right tibial external rotation (RTER), the right tibial internal rotation (RTIR), the left tibial external rotation (LTER) and the left tibial internal rotation (LTIR) have been made for all participants by using the MVARTS system described above. Figure 6 shows the distribution of all measurements. It should be noted that the whole data have been sorted in a way to train and test the whole data set in equal amounts. The first half of data shows the patterns used to train the Neural Networks and the second half being used as testing set. The four parameters were mainly used as output parameters and also as input parameters as being additional input data to physical factors that are provided in Figure 7.

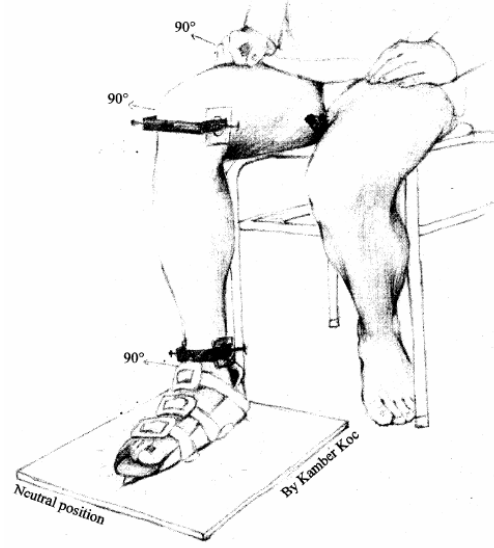

Figure 2. MVARTS system with radiological markers

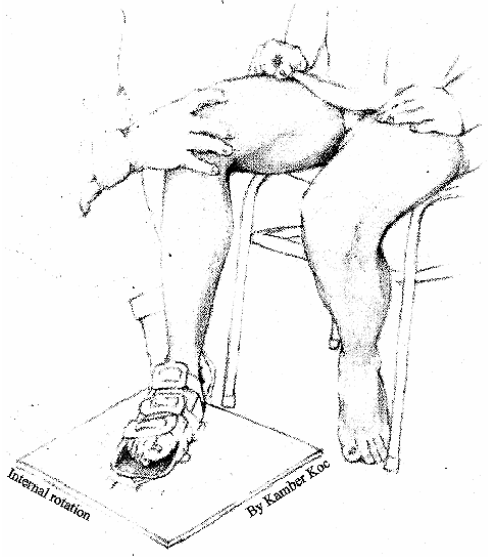

Figure 3. Measurement of medial tibial and subtalar rotations in MVARTS system

Another data set comprised of four physical parameters related to the participants corresponding to the patterns in Figure 6 in the same order. These four parameters including gender, age, weight and height were mainly used as input parameters in the design of Neural Networks that will be described in the next section. Figure 7 shows the distribution of these physical factors. The whole data was sorted accordingly by following the order in the tibial motion parameters provided in Figure 6. The next section describes the assessment of tibial rotations using ANNs.

\subsection{Artificial Neural Networks for the Assessment of Tibial Rotations}

ANNs have been implemented for solving problems in many areas ranging from medical imaging [5] to prediction of diseases based on certain medical factors [6]. However, the assessment of tibial rotation parameters using ANNs is new. This study 
makes a thorough study of some physical factors and examines their relation with the tibial motion parameters based on ANNs.

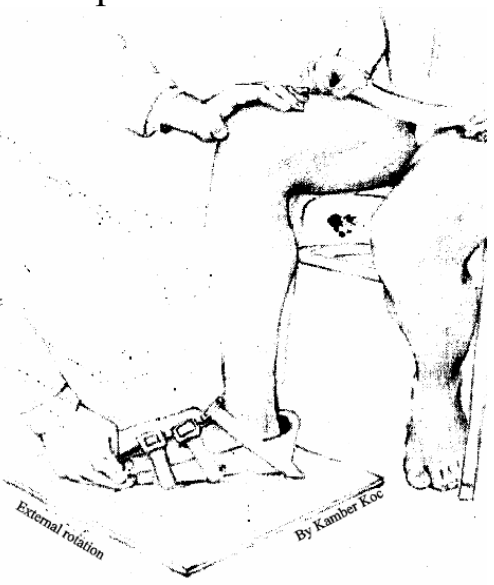

Figure 4. Measurement of lateral tibial and subtular rotations in MVARTS system

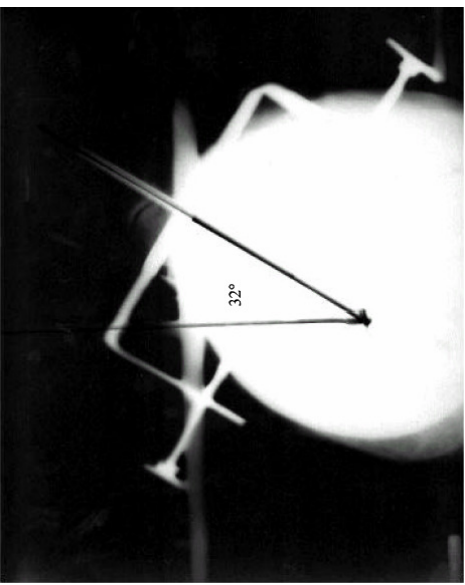

Figure 5. Calculation of tibial rotation on X-Ray Film

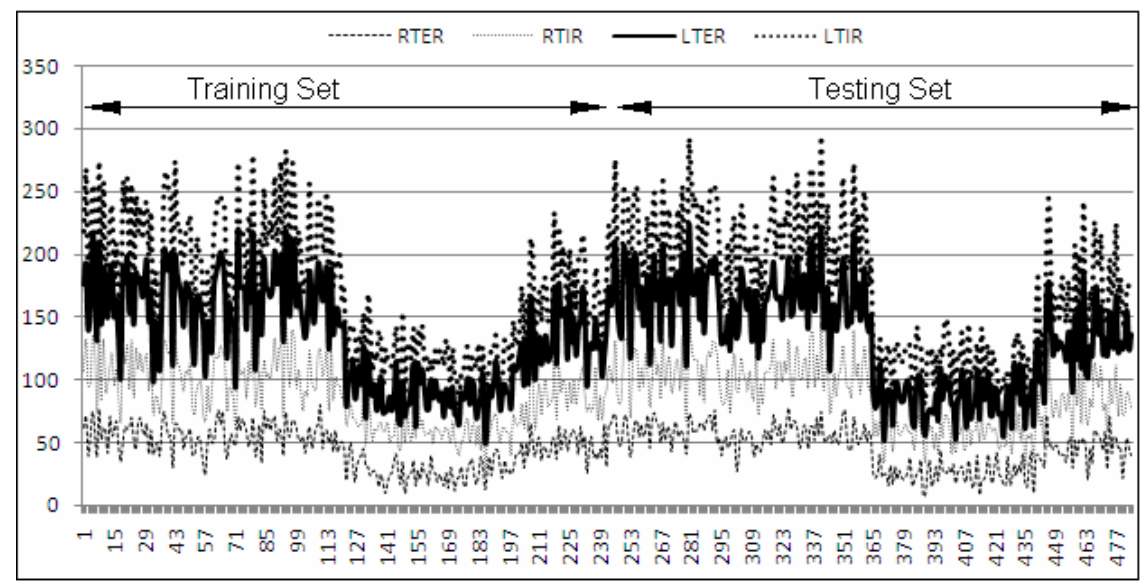

Figure 6 Tibial Motion Measurements for all participants

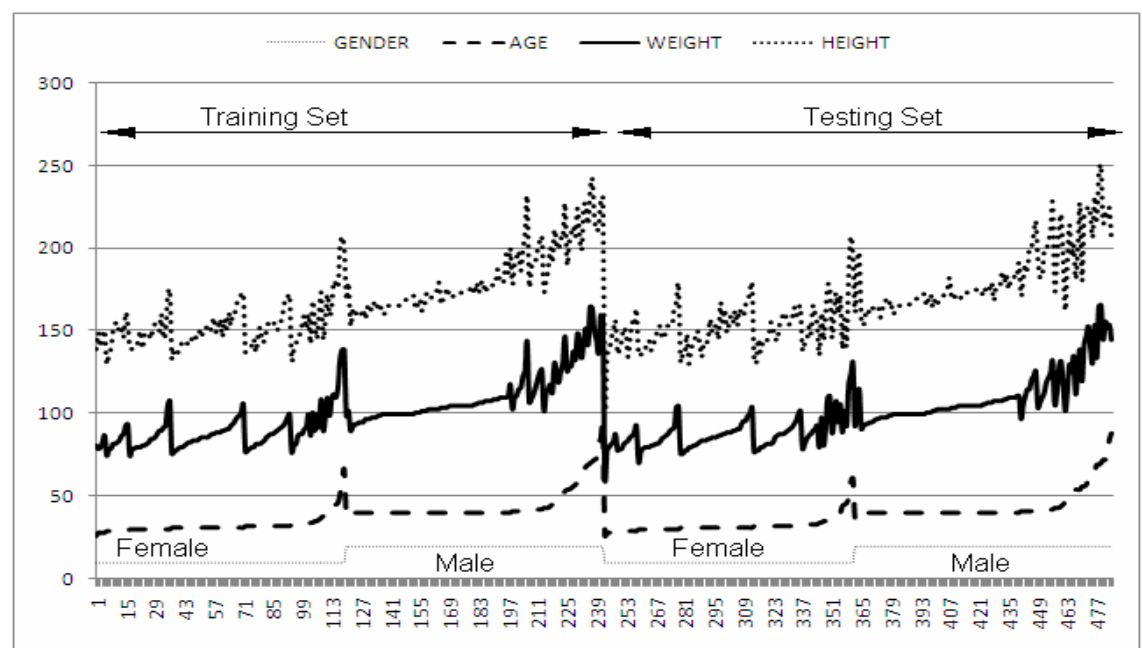

Figure 7 Physical Factors for the Corresponding Tibial Motion Parameters 
ANNs have been popular recently in medicine due to their feasibility to model and later predict the patterns existing in medical applications. The ANN model composes of a large number of interconnected units divided into input, output, and hidden nodes. A single processing unit merely sums up the weighted activation on its inputs, transforms this sum according to an activation function, and passes the resulting function to its output. Therefore, in general terms, information processing in neural networks consists of the units transforming their input into some output, which is then modulated by the weights of connections as inputs to other units. Learning in these systems is defined in terms of the total adjustments of the weights continuously so that the network's output tends toward the desired output without involving changes to the structure of the network. Neural networks are trained by specifying some constraints such as learning parameters, network structure, and training examples. When learning is complete, or stopped at an adequate level, knowledge is said to be represented by the optimized connection weights among processing units in the entire network.

Unlike traditional expert systems where a knowledge base and necessary rules have to be defined explicitly, neural networks do not need rules. They, instead, generate rules by learning from given examples. This makes ANNs general purpose classification tools to be used in pattern recognition and classification systems. Neural networks provide a closer approach to human perception and recognition than traditional computing. When inputs are noisy or incomplete, neural networks can still produce reasonable results. Neural networks are used successfully in many areas such as Natural Language Processing, Speech Processing, Data Compression, Computer Security, Image Recognition (Optical Character Recognition, Texture Classification, Handwriting Recognition, Target Classification, Industrial Inspection), Optimization problems, Signal processing (Prediction, System Modeling, Noise Filtering etc.), Financial and Economic Modeling and Control Systems. Possibility and ease of the use in many areas make ANNs an ideal solution for many applications.

The ANN model with Back Propagation algorithm was used in this study [7]. The model consists of three layers namely input layer, hidden layer and output layer. The input layer composed of 4 physical parameters which are considered to have a relation with 4 tibial motion parameters under the study. The 4 physical factors were gender, age, weight and height. Figure 8 shows the adopted ANN for the prediction of the effect physical factors on the tibial rotations. The first design of ANN consists of mainly physical factors in the input layer to predict the tibial motion parameters. The second design of ANN has an additional input neuron in the input layer to represent one of the tibial motion parameters. The hidden layer was found to be optimum with 4 processing elements based on trial and error.

As reported in the following section, there is significant relation existing between some of the parameters while the relation is relatively weak for some. The data pattern consisted of gender, age, weight and height values as well as left and right external and internal tibial rotations taken from 484 healthy subjects. Initially, the four physical factors alone were used to investigate the effect on the four rotations. The second ANN consisted of one additional tibial motion parameter as additional input to physical factors. The Neural Networks were run separately for each case. The existence of relations among the different parameters is reported in the following section. 

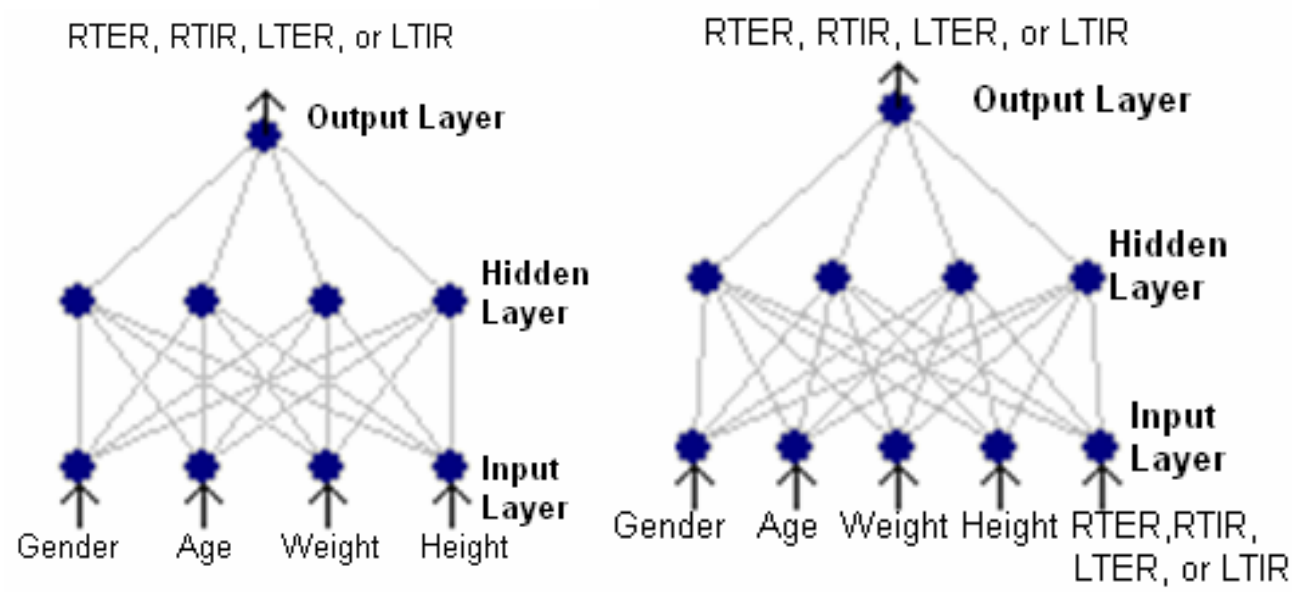

Figure 8. The adopted ANN network structures (a) Only Physical Factors, and (b) Physical Factors plus one tibial motion measurement

\section{RESULTS}

Most of the subjects examined in this study are under the age of 30. RTER, RTIR, LTER and LTIR stand for the right tibial external rotation, the right tibial internal rotation, the left tibial external rotation, the left tibial internal rotation, respectively.

Here 484 subjects (238 females and 246 males) were considered. Whilst the females are in the range of 16-56 years, the males are between 16 and 74 years old. For both extremities; the amount of rotations for the female subjects is more than the male subjects.

The whole data set was divided into two sections as training and testing data for validation purposes. The testing data was never presented to Neural Networks and they were mainly used for testing the performance of the Networks. Two different designs of networks have been trained for an average of 20,000 epochs. Table 1 shows the tabulated results for both networks with a total of 16 configurations. Network Type $A$ has physical factors only in the input layer whereas Network Type $B$ contains an additional parameter taken from one of tibial motion measurements or assessments (see Figure 8 (a) and Figure 8 (b)). The GAWH in table represents four physical factors as gender, age, weight, and height. Predicted outputs for RTER, RTIR, LTER, and LTIR are ordered according to performance criteria (mainly correlation coefficients calculated by recalling the network on testing data). As illustrated in Table 1, the tibial motion RTER has very good predictive performance even with only four physical factors as being 0.78 . Prediction performance for LTER is also good with 0.77 for GAWH only. Since main objective of the study is to assess tibial motion parameters by minimum effort due to their difficulty of measurement, the network type $A$ is the most preferred one. However, Networks may be utilized on predicting two tibial motions of RTER (Configuration 3) and LTER (Configuration 10) by using GAWH patterns only and then using two predicted results (RTER and LTER) to assess the remaining parameters of RTIR and LTIR. By measuring only the RTIR parameter, the remaining LTIR may be predicted from Configuration 13 with a high correlation coefficient of 0.74 . Shaded rows in Table 1 show the tibial rotation predictions based only on physical factors of GAWH (network type $A$ ). 
After the training and testing of data sets individually a significant relation was discovered between 4 physical factors (GAWH) and right external tibial rotation (RTER) as illustrated in Figure 9. The graph shows the actual results of RTER in comparison to predicted ones. It should be noted that the results were obtained from testing data which means the performance from training data was much higher. The correlation coefficient was calculated as 0.78 for the four input factors and the related RTER value.

Table 1 Performance Results from Both Networks for Assessment of Tibial Motions

\begin{tabular}{ccccc}
\hline Config. No. & Network Type & Inputs & Predicted Output & Correlation Coefficient \\
\hline 1 & B & GAWH+LTER & RTER & 0.88 \\
2 & B & GAWH+LTIR & RTER & 0.80 \\
3 & A & GAWH & RTER & 0.78 \\
4 & B & GAWH +RTIR & RTER & 0.74 \\
\hline 5 & B & GAWH+LTIR & RTIR & 0.59 \\
6 & B & GAWH+LTER & RTIR & 0.46 \\
7 & A & GAWH & RTIR & 0.34 \\
8 & B & GAWH+RTER & RTIR & 0.24 \\
\hline 9 & B & GAWH+RTER & LTER & 0.84 \\
10 & A & GAWH & LTER & 0.77 \\
11 & B & GAWH+RTIR & LTER & 0.75 \\
12 & B & GAWH+LTIR & LTER & 0.61 \\
\hline 13 & B & GAWH+RTIR & LTIR & 0.74 \\
14 & A & GAWH & LTIR & 0.42 \\
\hline 15 & B & GAWH+RTER & LTIR & 0.39 \\
16 & B & GAWH+LTER & LTIR & 0.38 \\
\hline
\end{tabular}

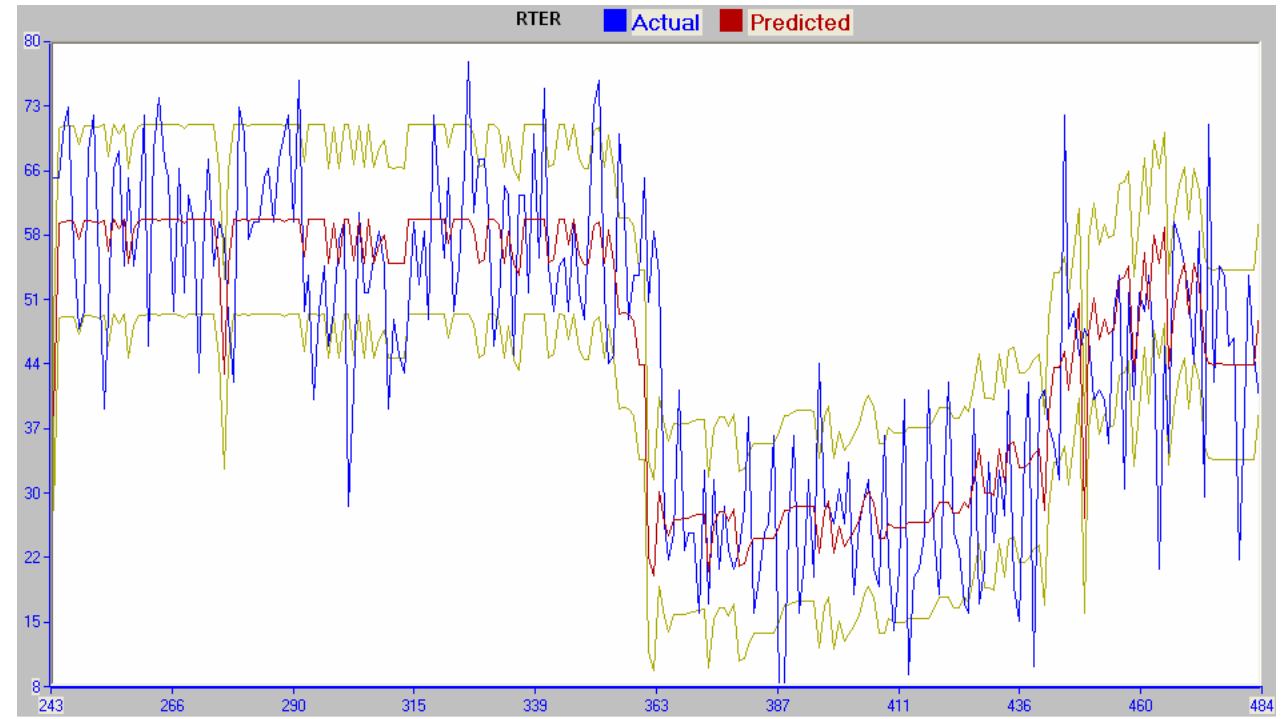

Figure 9. Comparison of Actual and predicted ANN values for RTER using Network Type $A$ (Configuration 3)

The left side of the graph contains the RTER values for female while the right side refers to male subjects. A very interesting and significant result was obtained when the remaining tibial rotational factors were predicted from ANN model. Figures 10, 11, 12 and 13 show the RTIR, LTER, and LTIR predictions, respectively. The neural 
network model produced as good as the RTER when predicting the relation between the four physical factors and LTER (configuration 10). Prediction of RTIR (configuration 7) and LTIR (configuration 14) by using only GAWH did not produce good results as giving lower correlation coefficients of 0.34 and 0.42 , respectively. The prediction of tibial assessments is based only on physical factors only in Figures 10, 11 and 12 (network type $A$ ) whereas network type $B$ is adopted to predict the LTIR (see Figure 13).

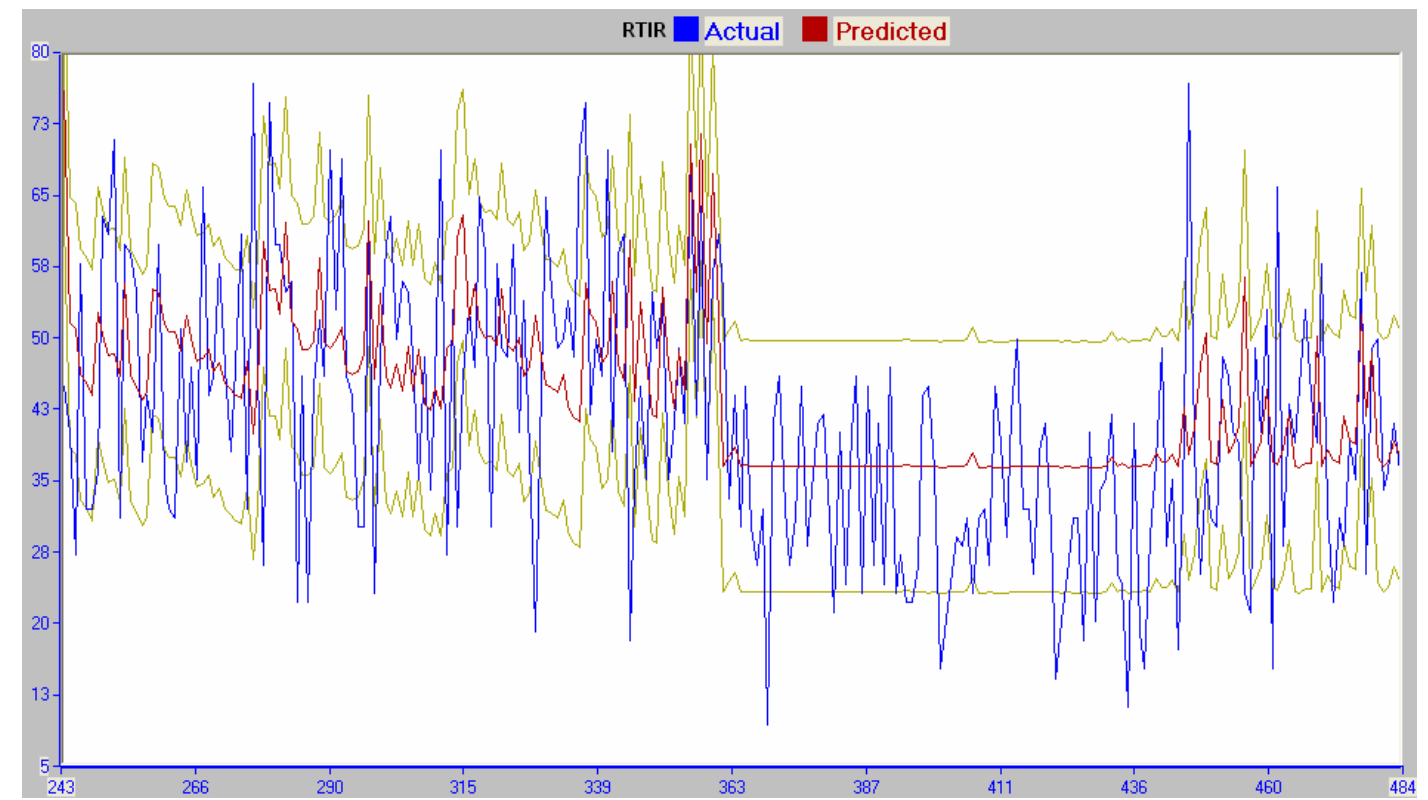

Figure 10. Comparison of Actual and predicted ANN values for RTIR using Network Type $A$ (Configuration 7)

Originality of this work lies on the design of ANNs where not only the physical factors (network type $A$ ) are taken into consideration for predicting the tibial rotations but also using the predicted patterns to predict other tibial motions (network type $B$ ). As an example, both networks in Figure 12 and Figure 13 are used to predict the tibial rotation of LTIR. However, prediction performance in Figure 13 is much higher $(0.74$ vs 0.42 ) when using a predicted parameter of RTIR as an additional input to GAWH factors compared to Figure 12 where only four physical factors are used for predicting RTIR.

The most significant finding from all results is that there is very strong relation between RTER/LTER and physical parameters. On the other hand, this relation is not as strong as when taking into consideration internal rotations (RTIR/LTIR). This may be explained by two different phenomena. First, the external usages of both feet are more effective and natural in daily life, and hence making external rotations easier to identify.

Secondly, more data may be required to train the neural networks for detecting internal rotations more reliably. In this study, RTER and LTER predictions give better results than the rest of the rotational predictions. The predictability of the internal rotations is clearly lower than the external rotations. Despite the lower performance of prediction of internal tibial rotations by relying only physical factors, a higher 
performance may be achieved by combining physical factors as suggested in this work with the predicted results (see Figure 13).

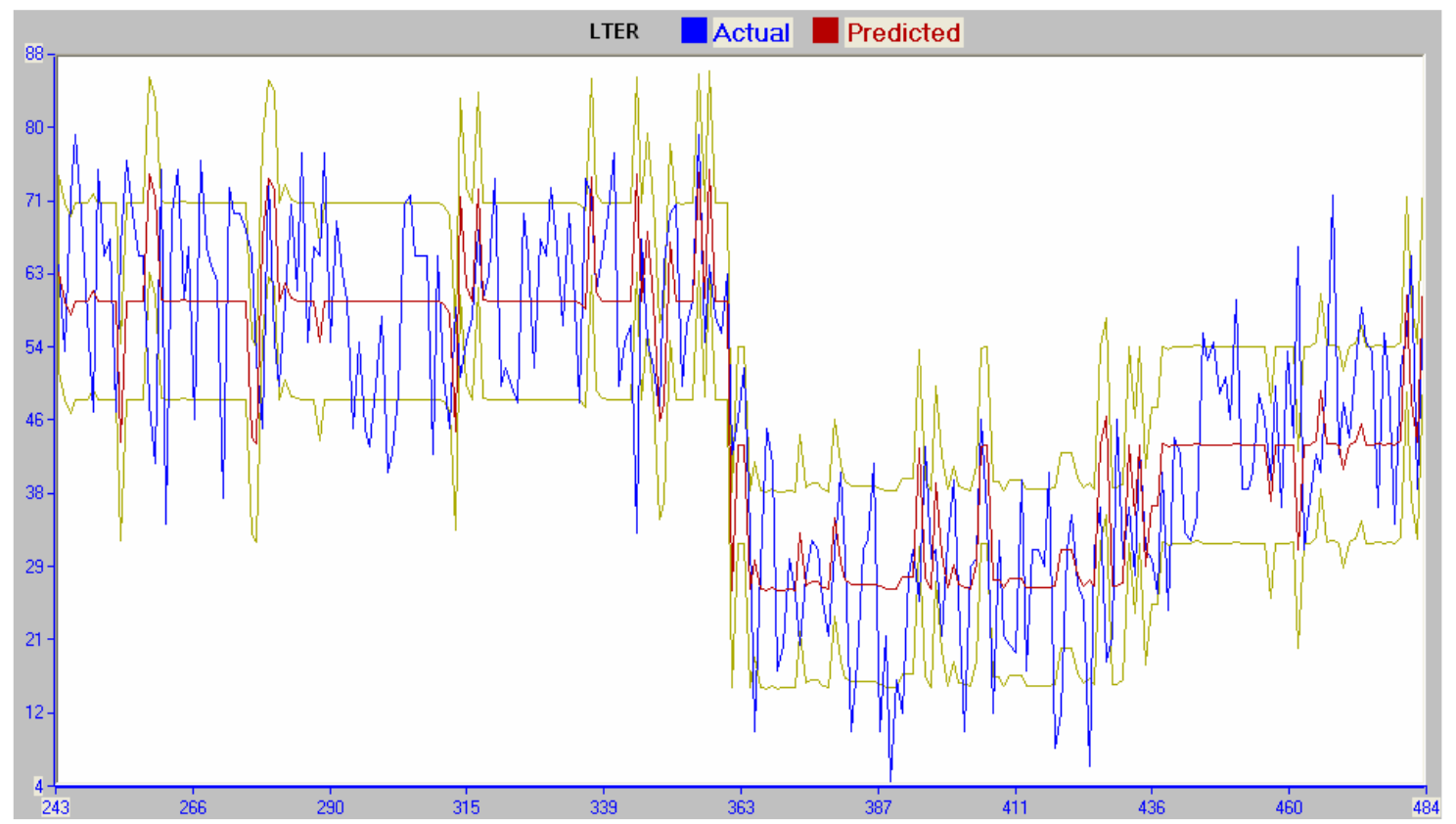

Figure 11. Comparison of Actual and predicted ANN values for LTER using Network Type $A$ (Configuration 10)

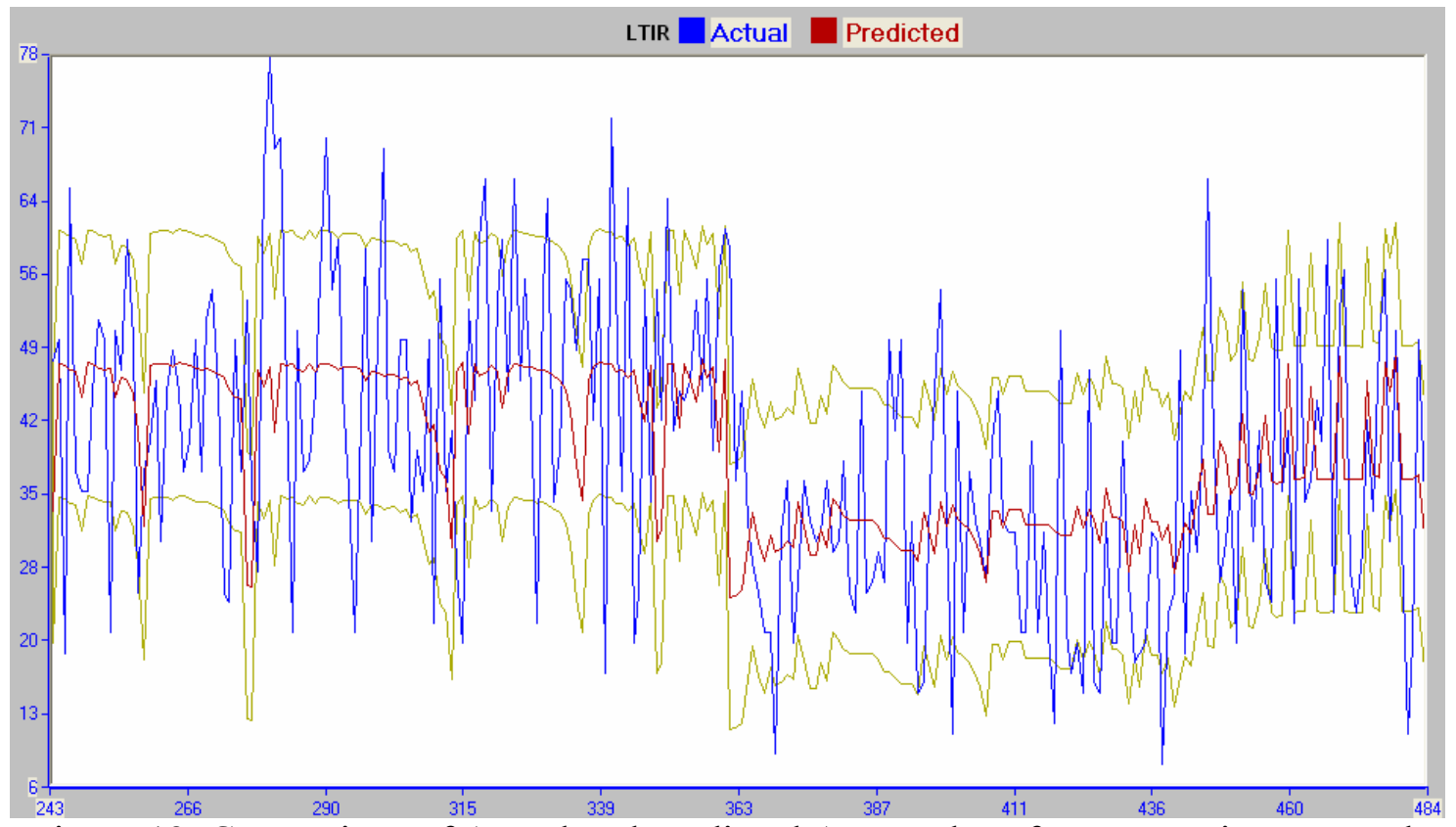

Figure 12. Comparison of Actual and predicted ANN values for LTIR using Network Type $A$ (Configuration 14) 


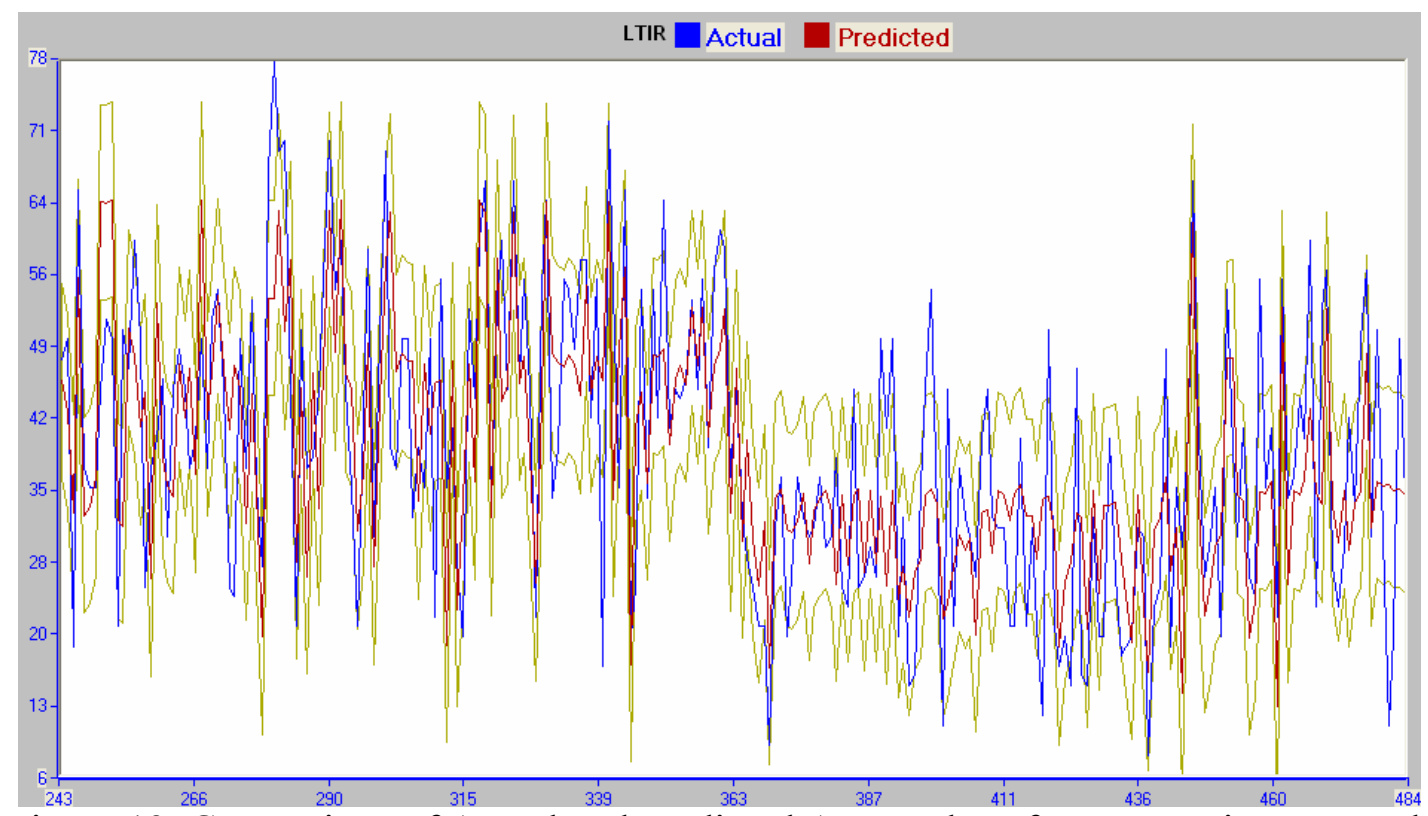

Figure 13. Comparison of Actual and predicted ANN values for LTIR using Network Type $B$ (Configuration 13)

\section{DISCUSSION AND CONCLUSION}

The aim of this study was to model the effect of physical factors on tibial rotations and hence to assess the tibial motion based on these parameters. To this end, the results belonging to 484 healthy subjects are analyzed using the ANN. The outputs (RTER, RTIR, LTER and LTIR) are predicted with the correlation coefficients as 0.78 , $0.59,0.77$ and 0.74 respectively.

In literature, since there is a strong relation reported between the tibial motion and certain knee injuries $[8,3]$, it is believed to be important to measure the external and internal tibial rotations during daily examination. Therefore, this study focused on prediction of the effects of the physical factors on the passive tibial rotations. A significant relation was discovered between 4 physical factors and right/left external tibial rotations. Performance increases have been discovered by also taking the predicted results into the networks as additional parameters. It is believed that more physical factors may be useful to predict the other 2 tibial rotations with high accuracy as in the prediction of the right external and left external tibial rotations. Therefore, a further research may be carried out for such purpose.

All studies carried out in literature points out the necessity and importance of the measurement of the tibial rotations. This cause directed us to predict the relation between the physical factors and the tibial rotations.

This research was limited to a population subjects who did not have knee injuries. The current research would be better if Q-angle and knee muscles' strength were included. For further research, attention can be paid on describing the physical factors by looking for differences between healthy individuals and those with different knee pathologies. 
Acknowledgements- The authors thank Dr. A. Cimbiz (Dumlupinar University, Health Institution of Higher Education, Department of Physical Therapy, 43100, Kutahya, Turkey) for providing the data and his helpful comments.

\section{REFERENCES}

1. J. C. Küpper, B. Loitz-Ramage, D. T. Corr, D. A. Hart and J. L. Ronsky, Measuring knee joint laxity: A review of applicable models and the need for new approaches to minimize variability, Clinical Biomechanics, 22(1), 1-13, 2007

2. L. E. DeFrate, H. Sun, T. J. Gill, H. E. Rubash and G. Li, In vivo tibiofemoral contact analysis using 3D MRI-based knee models, Journal of Biomechanics, 37, 14991504,2004

3. J. Hamill, B. T. Bates and K. G. Holt, Timing of lower extremity joint actions during treadmill running, Medicine \& Science in Sports \& Exercise, 24(7), 807-813, 1992

4. A. Cimbiz, U. Cavlak, M. Sari, H. Hallaceli and F. Beydemir, A new clinical design measuring the vertical axial rotation through tibial shaft resulting from passive knee and subtalar joints rotation in healthy subjects: a reliability study, Journal of Medical Science, 6(5), 751-757, 2006

5. A. S. Miller, B. H. Blott and T. K. Hames, Review of neural network applications in medical imaging and signal processing, Journal of Medical and Biological Engineering and Computing, 30(5), 449-464, 1992

6. T. Nguyen, R. Malley, S.H. Inkelis and N. Kuppermann, Comparison of prediction models for adverse outcome in pediatric meningococcal disease using artificial neural network and logistic regression analyses, Journal of Clinical Epidemiology, 55(7), 687-695, 2002

7. R. P. Lippman, An introduction to computing with neural nets, IEEE ASSP Magazine, 4, 4-22, 1987

8. S. L. James and D. C. Jones, Biomechanical aspects of distance running injuries, Biomechanics of Distance Running, ed P. R. Cavanagh. Human Kinetics Books, Champaign: Illinois, 1990 